\title{
EL COMPORTAMIENTO DEL CONSUMIDOR DE LOS APLICATIVOS MÓVILES PARA RESTAURANTES EN EL CONTEXTO DE LA PANDEMIA DEL COVID-19
}

\section{CONSUMER BEHAVIOR OF MOBILE APPLICATIONS FOR RESTAURANTS IN THE CONTEXT OF THE COVID-19 PANDEMIC}

\author{
Valentina Gomes Haensel Schmitt ${ }^{1}$, Daniela Mercedes Massey Tapia ${ }^{2}$, Nicolas \\ Augusto Santa Gadea Massucco ${ }^{3}$
}

\begin{abstract}
Palabras clave: Resumen aplicativos La presente investigación tuvo por objetivo analizar el móviles, comportamiento del consumidor de los aplicativos móviles de comportamiento restaurantes de Lima, Perú en el 2020, estando enfocado en el del consumidor, contexto de la pandemia del COVID-19. El método ha sido delivery, restaurantes, COVID-19. cuantitativo, correlacional, con la recolección de datos durante el segundo semestre de 2020. La muestra estuvo compuesta por 385 personas. El estudio demostró la relación entre las variables, debido a características como: ubicuidad, personalización y riqueza de la información; que influyen en la motivación y frecuencia de compra. Además, se verificó la importancia de la "nueva seguridad".

Códigos JEL: M31, D15
\end{abstract}

\begin{abstract}
Keywords:
mobile applications, consumer behavior, delivery, restaurants, COVID-19.
\end{abstract}

\begin{abstract}
The objective of this research was to analyze the consumer behavior of the mobile applications of restaurants in Lima, Peru in 2020, being focused on the context of the COVID-19 pandemic. The method has been quantitative, correlational, with data collection during the second semester of 2020. The sample consisted of 385 people. The study demonstrated the relationship between the variables, due to characteristics such as: ubiquity, personalization and richness of information; that influence the motivation and frequency of purchase. In addition, the importance of the "new security" was verified.
\end{abstract}

\footnotetext{
${ }^{1}$ Universidad de Lima, (Perú).

E-mail: vschmitt@ulima.edu.pe

${ }^{2}$ Universidad de Lima, (Perú).

E-mail: 20162245@aloe.ulima.edu.pe

${ }^{3}$ Universidad de Lima, (Perú).

E-mail: 20162530@aloe.ulima.edu.pe
} 


\section{INTRODUCCIÓN}

El creciente uso de internet se ha visto facilitado por la difusión del comercio electrónico vía web, las aplicaciones de comercio móvil para compras en línea, pagos móviles para diferentes categorías de productos, incluidos los restaurantes (Shang y Wu, 2017). En la actualidad, las empresas cada vez más consideran el comercio electrónico como uno de sus canales principales de venta. En el Perú, según la Cámara Peruana de Comercio Electrónico (CAPECE), en 2019 el ecommerce registró un crecimiento del $30 \%$, una de las tasas más alta de la región, pero se mantuvo en el sexto lugar en Latinoamérica en cuanto al volumen de ventas en este canal (CAPECE, 2019).

El incremento de usuarios de smartphones, propició el desarrollo de una nueva rama del ecommerce, exclusivamente para hacer transacciones vía teléfonos móviles, denominada m-commerce - o comercio móvil -, que permite al consumidor realizar sus compras desde el teléfono y es un canal atractivo para el perfil actual del consumidor digital. En el Perú se estima que el $88 \%$ de los compradores en línea utiliza el smartphone como principal dispositivo para realizar compras, y del total de peruanos que utilizan aplicativos de delivery, el $92 \%$ lo hace para realizar pedidos de comida (Ipsos, 2019a). Además, en 2019 hubo 3.6 millones de compradores en línea en el Perú urbano y el smartphone fue el principal medio para realizar compras en línea (Ipsos, 2019b). Respecto a las ventas totales del comercio electrónico en el país durante el mismo período, los aplicativos móviles representaron el $42 \%$, y la categoría de alimentos y restaurantes fue la segunda más relevante para este canal, generando el $22 \%$ de sus ventas (CAPECE, 2019).

Los efectos de la pandemia del COVID-19 han proporcionado un crecimiento mayor al previsto para el m-commerce, principalmente debido a las restricciones para compras en lugares físicos y al creciente temor de contagio de la población. Durante el 2020, debido a la coyuntura, los restaurantes han tenido que adaptarse a la "nueva normalidad", que involucró a un nuevo perfil de consumidor y a las restricciones del gobierno para la atención en locales físicos, como el aforo máximo de los establecimientos comerciales (El Peruano, 2020). Con eso, se generó la necesidad de adaptación de los negocios a las nuevas necesidades y tendencias de los consumidores. Ese proceso de adecuación es producto de los cambios tecnológicos vitales para la sostenibilidad de los negocios (Thihas y Thangavelan, 2018).

Por lo tanto, el objetivo de la presente investigación fue analizar el comportamiento del consumidor de los aplicativos (mcommerce) de restaurantes de Lima, Perú en el 2020. Al final, el estudio permite comprender la relación entre el m-commerce y el comportamiento de consumo bajo el contexto de pandemia, el perfil del consumidor y los aspectos relevantes bajo el contexto de "nueva normalidad". El articulo está estructurado en: Bases teóricas; Metodología utilizada; Resultados de la investigación; Discusión y, Conclusiones y recomendaciones del estudio.

\section{REVISIÓN DE LITERATURA}

\section{El comportamiento del consumidor digital en el contexto de la pandemia del COVID-19}

El comportamiento del consumidor involucra un proceso de decisión a través del cual los consumidores eligen o rechazan productos $\mathrm{y}$ servicios con el objetivo de satisfacer sus necesidades (Schiffman y Lazar, 2010). Para comprender el comportamiento del consumidor se puede tener de referencias aspectos como (Kotler y Armstrong, 2017): a) el cultural, que abarca los conocimientos, rituales, costumbres y otros hábitos que tenga el consumidor por parte de su entorno; b) el social, que involucra los grupos de referencia, que pueden llegar a tener una influencia positiva o negativa sobre las actitudes o conductas de los consumidores; c) el personal, dentro de este concepto se deben considerar diversos factores tales como la edad, el ciclo de vida, la ocupación, el estilo de vida y la personalidad y/o el autoconcepto del consumidor; d) el psicológico, compuesto de los factores internos tales como la motivación, la percepción, el aprendizaje y las actitudes y creencias, que influyen en la decisión de compra. 
Respecto al comportamiento del consumidor digital, se pueden establecer cuatro tipos (Abuladze y Todua, 2011): (a) los simplificadores, que son usuarios de internet impacientes pero que generan ganancias, pasan un par de horas al mes en línea y la mitad de las transacciones en línea son generadas por ellos; (b) los surfistas, que son navegadores de internet intensificados, que pasan el $32 \%$ del tiempo en línea y navegan cuatro veces más en diferentes páginas en comparación con otros usuarios; (c) los conectores, que son usuarios nuevos en internet y menos probables que compren; (d) los compradores de gangas o "bargain shoppers", adictos a la información, ofertas, promociones. Utilizan internet para unirse a comunidades virtuales y para informarse sobre productos o servicios.

Debido al contexto del COVID-19, se empieza a crear un nuevo consumidor y por ende, nuevos comportamientos de compra. Un ejemplo es el incrementó de $48 \%$ en el uso de aplicaciones para delivery durante el 2020 (McKinsey, 2020). Esto se relaciona con las crecientes preocupaciones del consumidor peruano por cuidar a su familia/amigos y a sí mismo (80\%); ya que, se reduce el contacto con otras personas (McKinsey, 2020). Es importante resaltar que, el comportamiento del consumidor digital varió en el transcurso de la pandemia distinguiéndose dos etapas: "cuarentena" y "reinicio de actividades económicas". En los consumidores entre 16-24 años, las compras a través de aplicativos y páginas web se incrementaron en un $9 \%$ en el reinicio de las actividades económicos en comparación con la cuarentena; en los de 25-34 años, hubo un crecimiento del 13\%; de 35 a 44 años, 11\%; de 45-54 años, 15\% y, de 55 años a más, 7\% (Datum Internacional, 2020).

Con relación a los principales productos que compraron los consumidores peruanos durante la cuarentena se encuentran los siguientes: alimentos (alimentos y bebidas para la alacena/restaurantes) con un $65 \%$, moda con un $36 \%$, equipamiento del hogar con un $34 \%$, y farmacia con un 33\% (Ipsos, 2020). Por último, los pedidos de restaurantes y supermercados representaron las compras por internet que más desconfianza y temor de contagio generaron en los usuarios, por lo que el $80 \%$ de usuarios del estudio "Perfil de los peruanos conectados" (2020), mencionó que no compraría a marcas que no sigan los protocolos de bioseguridad para evitar contagios.

\section{Comercio móvil}

Los aplicativos móviles están insertados en el contexto del comercio electrónico. Por lo tanto, para comprender el proceso de compra es fundamental saber que el e-commerce tiene ocho dimensiones principales, las cuales son (Laudon y Guercio, 2013): 1. La ubicuidad, que permite al consumidor realizar transacciones desde cualquier lado a su conveniencia y, haciendo uso únicamente de internet; 2. El alcance global, la tecnología permite que se amplíe la cobertura de clientes alrededor del mundo; 3. Los estándares universales, existe una base tecnológica común, económica y global para que utilicen las empresas; 4. La riqueza de la información, por la comunicación online a través de video, audio y texto para mejorar la experiencia del cliente, sin necesidad de realizar una venta directa; 5. La interactividad, la tecnología del comercio electrónico depende de la interacción con el cliente y de cómo desea la empresa que el cliente viva su experiencia; 6. La densidad de la información, los costos de información, almacenamiento se reducen; 7. La personalización y adecuación, que permite personalizar la experiencia de compra, tal cual como en un canal físico; 8 . La tecnología social, compuesta por la imagen y reputación de las empresas se ven afectadas por las redes sociales y su impacto en los usuarios. Además de estas características compartidas, los aplicativos móviles tienen tres específicas: conveniencia, localización (Clarke, 2001) y eficiencia en las transacciones (Nayyar, 2015). Cabe agregar que, la ubicuidad se relaciona directamente con la conveniencia y tiene un impacto positivo en la localización, ya que el usuario puede acceder a ofertas y realizar pedidos sin importar su ubicación (Bergström et al., 2015). Además, las transacciones a través de los aplicativos móviles son considerablemente más rápidas en comparación con aquellas realizadas desde una 
computadora, que requiere de un gran número de clics para ingresar a la página de la empresa ofertante (Nayyar, 2015).

La principal característica de las aplicaciones móviles que permite conectar emocionalmente con el consumidor es la personalización, que se refiere a adecuar la aplicación a sus intereses y preferencias (Mc Lean et al., 2018). La ubicuidad y conveniencia que brindan los aplicativos móviles son los principales factores por los cuales los clientes incorporan este canal a su vida cotidiana (Ashraf et al., 2016). Sin embargo, a pesar de las características y beneficios únicos que los aplicativos móviles brindan a los usuarios, este canal no está exento de retos y desafíos para las empresas. Por ejemplo, el tamaño de la pantalla del celular limita considerablemente los gráficos, imágenes y archivos que pueden ser presentados, además, el uso de la interfaz del aplicativo puede llegar a ser difícil de aprender para usuarios nuevos al canal (Nayyar, 2015). Por otro lado, un aspecto primordial, es que los usuarios consideran el factor del oportunismo, que viene a ser la percepción de que la empresa no ofrece toda la información y características de los productos que vende, como un aspecto que impacta de forma negativa en su lealtad hacia la marca, pero también hacia todo el canal (Jiménez et al., 2016).

\section{Comportamiento y actitudes del consumidor de aplicativos móviles}

Para comprender la relación entre los aplicativos móviles y el comportamiento del consumidor, es importante resaltar la influencia de la motivación y de la experiencia en el proceso de decisión de compra en línea. En relación con el primer factor, las empresas se deben enfocar en satisfacer las necesidades existentes de los clientes, a través de promociones, precios atractivos, y principalmente con un amplio catálogo de productos, que incentive la compra en línea, en vez de las compras en tiendas físicas. Por otro lado, para la experiencia del consumidor, la empresa debe evitar cometer errores en las transacciones, que pueden reducir su confianza y también deben encargarse de que su plataforma virtual sea amigable y fácil de utilizar (Mercado et al., 2019). Otro factor que incrementa la motivación de compra es la conveniencia que brindan los aplicativos, además de las diferentes opciones que pueden evaluar los clientes, lo que permite una mejor experiencia de compra, ya que se reduce la asimetría de información entre el canal y el cliente (Hafi, 2020).

Asimismo, en el proceso de aceptación de este canal por parte de los clientes se encuentran aspectos como: (a) las experiencias favorables de manera digital, la percepción de utilidad y facilidad de uso de este canal de venta; (b) la conectividad a la plataforma digital, que tienen gran influencia en la calidad de la experiencia que percibe el cliente cuando realiza la transacción, lo que se refleja en la intención de uso en el futuro. Luego, es importante que las empresas busquen desarrollar el hábito en los consumidores respecto a las compras en línea (Hubert et al., 2017). Sin embargo, la desmotivación hacia la compra a través de internet se puede explicar a partir de tres factores, que son: la seguridad de la información del usuario, la intangibilidad del servicio y los errores de la plataforma (Jiménez y Martin, 2007).

Los factores de motivación y experiencia del usuario explicados anteriormente pueden ser analizados a profundidad con un marco conceptual denominado modelo de información (i- model) que propone que la información con la ayuda de la tecnología puede proveer beneficios a las personas y la sociedad en su conjunto. Este modelo cuenta con cuatro componentes fundamentales, que son los siguientes: información. tecnología, personas y sociedad (Zang y Benjamin, 2007). En el estudio de Du y Li (2019) se aplicó este modelo para analizar el comportamiento y la actitud de los usuarios ante el uso de aplicativo de móviles. Para el componente de personas, la utilidad percibida y la facilidad de uso de los aplicativos son claves, en el aspecto tecnológico, la calidad del sistema, el nivel de conectividad y la interfaz del aplicativo; en el aspecto de información, la calidad de la misma y la posibilidad de interacción con las empresas ofertantes; por último, en el aspecto social, la protección de la información de los usuarios y el cumplimiento de las normativas legales propias de las transacciones digitales, son fundamentales. 
Otro marco conceptual para entender la relación entre los aplicativos móviles y el comportamiento del consumidor es el propuesto por Lila Rajabion (2015), en donde se buscan establecer los principales factores que influyen en la decisión de los adultos jóvenes de adoptar tecnologías móviles. El primer concepto del modelo es la tecnofobia, que se relaciona con la incomodidad o baja disposición de las personas a utilizar la tecnología para la adquisición de bienes y servicios. La medición de esta variable puede servir para el proceso de segmentación, y así establecer los potenciales clientes que vayan a adoptar las nuevas tecnologías a su vida $\mathrm{y}$ aquellos que no (Sinkovics, Stöttinger, et al., 2002). La intangibilidad mental, que se refiere a la falta de claridad en la mente del consumidor en relación un producto, aunque este sea físicamente tangible. El nivel de intangibilidad mental se relaciona directamente con el riesgo percibido, específicamente, en el caso de productos, el grado de ambos se reduce e incrementa la posibilidad de que el cliente confíe en la empresa y finalmente adquiera el producto (Laroche, Bergeron, et al., 2001). Finalmente, hay la tendencia a la compra impulsiva, que está reflejada por la probabilidad de que un individuo realice compras involuntarias, inmediatas y directas (Jones, Reynolds, et al., 2003). Un ejemplo de lo que vienen a ser las compras por impulso, que son más frecuentes en el canal m-commerce por la facilidad de realizar transacciones (Rajabion, 2015).

Con respecto a los nuevos formatos de venta online, se verifica que los compradores priorizan factores como las políticas de retorno de productos y reputación de la empresa, en vez de los motivadores del canal retail tradicional, como las facilidades de parqueo, el servicio, la calidad, entre otros. Para los compradores digitales, los factores mencionados anteriormente, representan la razón por la cual están dispuestos a promocionar y recomendar una tienda en específico (Pan, Zinkhan, 2006). Además, el modelo presenta las dimensiones de confianza e intención del usuario. La confianza se relaciona con la tecnofobia, ya que establece el grado en el cual el consumidor confía en el canal mcommerce y en la seguridad de su servicio, determinando a futuro, la posibilidad de que el usuario tenga la intención de realizar transacciones a través de este canal. Por último, un hallazgo importante es que la adición de presencia social en el contexto del comercio electrónico aumenta la confianza del usuario, debido a que, por lo general, los sitios web carecen de interacción humana real (Gefen y Straub, 2004).

\section{El servicio de los restaurantes}

En lo que corresponde a la experiencia gastronómica de los restaurantes, los consumidores no solo buscan recibir productos y servicios, sino una experiencia completa (Kim et al., 2018). La experiencia en el restaurante debe incluir tres aspectos principales, que son: la calidad del producto, la calidad del servicio y un amigable entorno físico (Ryu et al, 2012). Estos conceptos conforman la experiencia completa que recibe el consumidor y que determinan su nivel de satisfacción y posibilidad de recompra. La calidad de la comida puede ser analizada a partir de diversos atributos, sin embargo, los siguientes son los más aceptados para medir la comida que ofrecen los restaurantes: forma de presentación del plato, sabor, frescura y temperatura de este; todos estos atributos son considerados como los aspectos tangibles de la calidad de la comida (Josiam et al., 2014).

Asimismo, en base a la escala SERVQUAL, se busca medir la percepción y expectativas de los clientes sobre la calidad del servicio que brindan las empresas (Parasuraman et al.,1988). Este concepto involucra aspectos como el envío del pedido, que es tercerizado por aquellos restaurantes que utilizan aplicativos móviles, y que por lo tanto no puede ser controlado por ellos, lo que en caso se realice de forma inadecuada, puede afectar el nivel de la calidad del servicio que percibe el consumidor final.

A su vez, el modelo Servicescape considera tres dimensiones del entorno para medir la calidad de este, que son: (a) condiciones del ambiente: ruido, aroma, música, iluminación, temperatura, entre otros; (b) espacio: distribución del local, muebles y equipamiento y (c) símbolos y señales: como la decoración, el diseño del local (Bitner, 
1992). Estos aspectos en su mayoría solo pueden ser entregados por los restaurantes en sus locales físicos, lo que limita la experiencia de los consumidores.

Finalmente, para entender la gestión de marketing en los restaurantes, es importante saber que existen diferentes clasificaciones, siendo la más utilizada a nivel global la propuesta por la National Restaurant Association (2010). La clasificación distingue entre cinco tipos de restaurantes, los cuales son: 1. Restaurantes de comida rápida, establecimientos en donde generalmente se realiza el pedido y se paga antes de consumir la comida. (fast food); 2. Restaurantes de comida rápida casuales, atractivos y cómodos establecimientos que sirven comida fresca, recién preparada, de calidad, de manera rápida; 3. Restaurantes familiares, el personal del restaurante provee de un buen servicio a los comensales, se les toma la orden una vez sentados en la mesa; 4. Restaurantes casuales, es un híbrido entre un restaurante familiar y uno exclusivo, que incluye una alta calidad de servicio y de comida, pero a un menor precio que un restaurante exclusivo; 5. Restaurantes finos o exclusivos, con alta calidad de servicio y de comida, buscando que el consumidor se siente cómodo y disfruta el ambiente del restaurante. El tipo de restaurante influye en el valor que se le brinda a cada uno de los aspectos de la experiencia mencionados anteriormente.

\section{METODOLOGÍA}

El enfoque utilizado para la investigación fue cuantitativo y de alcance de tipo correlacional, ya que se buscó relacionar una variable independiente (aplicativos móviles de delivery) y una dependiente (comportamiento del consumidor de restaurantes). Asimismo, la investigación es del tipo transeccional o transversal, debido a que la recolección de datos se realizó en un solo momento, en un tiempo único (Hernández, Fernández \& Baptista 2014), que fue el segundo semestre de 2020. En relación con el diseño muestral, se realizó un muestreo probabilístico, en base al estudio de Ipsos (2019a) sobre Compradores en línea del 2019, el cual estableció que el número de compradores en línea a través de smartphones en Lima fue de 1,450,592 personas. Como resultado se obtuvo un tamaño de muestra de 384 consumidores de restaurantes vía aplicativos de delivery durante el 2020.

Para la recolección de datos, se aplicó un cuestionario virtual vía Google Forms a 384 consumidores de restaurantes que han utilizado aplicativos móviles en Lima, Perú en el 2020. Este cuestionario consideró dos preguntas filtro para el usuario que viva en Lima y que haya utilizado aplicativos móviles de delivery para hacer pedidos de restaurantes durante el 2020. Asimismo, se presentaron 19 preguntas relacionadas con las variables de investigación comportamiento del consumidor y aplicativos móviles; dos preguntas enfocadas a la experiencia gastronómica que brindan los restaurantes a sus consumidores y una pregunta abierta para comentarios o sugerencias sobre el tema de la investigación. Del total de respuestas obtenidas que fueron 414, se consideraron 385 como válidas ya que cumplieron con las preguntas filtro planteadas.

Para el análisis de datos se utilizó el programa SPSS, que permitió el uso de diversos estadísticos y herramientas para el análisis de la información. Para determinar la validez del instrumento de recolección de datos, se utilizó el estadístico Alfa de Cronbach que tuvo un valor de 0.701 indicando que el instrumento de recolección es válido. Por último, para establecer si la dependencia se utilizó el estadístico Rho de Spearman, que resultó de 0,45 con un valor $\mathrm{p}=0,00<0.05$; por lo que se afirma que existe una correlación positiva media entre las variables comportamiento del consumidor y aplicativos móviles.

\section{RESULTADOS}

Respecto al perfil de los encuestados, la mayoría $(64,9 \%)$ tiene edades entre 18 y 25 años, $17,4 \%$ entre 26 y 30 años y 13,3\% entre 31 y 55 años. Asimismo, respecto al perfil del consumidor, se observó que el $29,1 \%$ de los encuestados tiene un ingreso promedio mensual entre 931 y 2000 soles, $18,4 \%$ entre 2001 y 3000 soles, $16,1 \%$ entre 3001 y 5000 soles, $14,8 \%$ menos de 930 soles, 10,6\% no tiene ingresos, $9,4 \%$ entre 5001 y 10000 soles y el $1,5 \%$ restante, tiene ingresos de 10001 soles en adelante. 
En lo que corresponde al comportamiento del consumidor de aplicativos móviles, se verificó que la mayoría (58\%) afirmó realizar compras por instrumentos de m-commerce al menos $1 \mathrm{vez}$ a la semana. En relación con si aprobaban las ofertas y promociones de los aplicativos de delivery, la mayoría de encuestados (76\%) se sintió al menos identificado. Por último, el $71 \%$ afirmó tener en su teléfono más de un aplicativo de delivery descargado.

Con respecto a los beneficios propuestos, la mayoría mencionó los siguientes: 1. el delivery gratis o barato que ofrecen los aplicativos $(86,2 \%)$; 2. los descuentos y promociones $(89,3 \%)$; 3. la variedad de restaurantes que ofrecen los aplicativos $(89,6 \%)$; 4. poder ver el estatus del pedido, la mayoría $(86,7 \%) ; 5$. la rápida respuesta del área de soporte de los aplicativos de delivery $(86,8 \%)$; 6. la seguridad que brindan los aplicativos de delivery $(93,5 \%)$.

Al buscar identificar si el público se había visto influenciado por sus grupos de referencia para realizar comprar por aplicativos de delivery, se verificó la mayoría de encuestados $(75,1 \%)$ consideró haber sido influenciado. Asimismo, se observó que la mayoría de encuestados $(66,3 \%)$ tiene un ticket promedio entre 21 y 60 soles y el $31,2 \%$ consume de 61 soles a más en los restaurantes.

Sobre los lugares de uso de los aplicativos de delivery, se verificó que el $37,5 \%$ lo tiene a su casa como el lugar preferido, el 30,6\% utiliza en las reuniones familiares o sociales, el $21,8 \%$ su centro laboral y el $10,1 \%$ su centro de estudios. Sin embargo, en ese aspecto es importante mencionar que en el Perú, desde el inicio de la pandemia del COVID-19, tanto reuniones como la asistencia a centros laborales y centros de estudio han sido restringidas.

Además, se verificó que las comunicaciones que reciben de los aplicativos de delivery como publicidad y promociones generan un aumento en su frecuencia de compra $(64,4 \%)$. En referencia a la sensación de seguridad al introducir información personal en los aplicativos de delivery, la mayoría de encuestados $(69,1 \%)$ estuvo totalmente de acuerdo y de acuerdo.
Respecto a la experiencia gastronómica en restaurantes, la mayoría de encuestados (66\%) consideró que la calidad de la comida es el aspecto más relevante de la experiencia gastronómica, mientras que el 12,9\%, consideró que los aspectos más importantes son el ambiente del local, la exclusividad del restaurante y el nivel de innovación de los platos. Finalmente, respecto al tipo de restaurante, se verificó que la mayoría de encuestados $(76,1 \%)$ prefirió los restaurantes de comida rápida, los de sushi/poke bowl, comida criolla/cebicherías y pollerías, mientras que el $21 \%$ tiene preferencia por los restaurantes de carnes y parrillas, de comida italiana y los chifas.

Adicionalmente, luego de revisar las estadísticas descriptivas y el análisis de la correlación entre las variables principales, que según el estadístico de Rho de Spearman resultó 0,451 ; se realizaron distintas tablas cruzadas con el fin de analizar si existe o no relación entre las dimensiones de las variables analizadas. Para eso, se utilizó el estadístico Chi-Cuadrado $\left(\mathrm{X}^{2}\right)$, para comprobar si son dependientes o independientes $\mathrm{y}$, el coeficiente de contingencia (C) para determinar el nivel asociación, en caso sean dependientes. Se tomó como referencia, un alfa $(\alpha)$ de 0,05 para el análisis de las pruebas de hipótesis.

En relación a las dimensiones edad e impacto de las comunicaciones en la frecuencia de compra, la mayoría de encuestados menor a 30 años considera que los distintos tipos de comunicaciones de los aplicativos de delivery (mensaje de texto, correos, contenido digital, entre otros) influyen positivamente en su frecuencia de compra. Esto se sustenta con la prueba chi cuadrado, donde se obtuvo un $\mathrm{p}=$ $0,011<0,05$; que permite rechazar la hipótesis nula y se concluye que hay relación entre las dimensiones analizadas, por lo que se afirma que son dependientes. Según el coeficiente de contingencia, se obtuvo un valor de 0,251 ; por esta razón, el nivel de asociación entre las dimensiones antes mencionadas es moderadamente bajo.

Sobre las dimensiones ocupación principal y lugar de uso del aplicativo de delivery, la mayoría de los estudiantes $(53.3 \%)$ prefieren su casa para 
hacer pedidos de delivery, los trabajadores independientes $(57,8 \%)$ y dependientes $(50,9 \%)$ prefieren su centro laboral; con respecto a los encuestados que tienen otra ocupación, el $47 \%$ prefiere su centro laboral y un $42 \%$ su casa. Esto se sustenta con la prueba chi cuadrado, donde se obtuvo un $\mathrm{p}=0,00<0,05$; y se establece que las dimensiones son dependientes. Según el coeficiente de contingencia, se obtuvo un valor de 0,270; por esta razón, se concluye que el nivel de asociación entre las dimensiones antes mencionadas es moderadamente bajo.

En los resultados de las dimensiones ingreso promedio mensual y ticket promedio de consumo por restaurante, la mayoría de encuestados con un ingreso promedio mensual menor a 2000 soles tienen un ticket promedio de consumo en restaurantes entre 21 y 60 soles; la mayoría de encuestados que tienen un ingreso entre $2001 \mathrm{y}$ 3000 soles $(63 \%)$, consumen entre 41 y 60 soles; por último, la mayoría de encuestados que tienen un ingreso entre 3001 y 5000 soles (66.1\%), consumen entre 41 y 80 soles. Con respecto a la prueba chi cuadrado, se obtuvo un $\mathrm{p}=0,00<0,05$; por lo tanto, se rechaza la hipótesis nula y se establece que son dependientes. Por otro lado, según el coeficiente de contingencia, se obtuvo un valor de 0,498; por esta razón, se concluye que el nivel de asociación entre las dimensiones antes mencionadas es moderadamente alto.

Con relación a la prueba chi cuadrado de las dimensiones pido delivery más de 1 vez a la semana y tengo descargado más de un aplicativo, se obtuvo un $\mathrm{p}=0,00<0,05$; por lo tanto, se rechaza la hipótesis nula y se establece que las dimensiones son dependientes. Por otro lado, según el coeficiente de contingencia, se obtuvo un valor de 0,409; por esta razón, se concluye que el nivel de asociación entre las dimensiones antes mencionadas es moderadamente alto.

\section{DISCUSIÓN}

Con referencia a los tipos de consumidores, el perfil que más resalta es el comprador de gangas, ya que la mayoría de encuestados (76\%) se sintió al menos identificado con ser un consumidor al que le gustan las ofertas y promociones que brindan los aplicativos de delivery; además, el
$71 \%$, se sintió al menos identificado con ser un consumidor que tiene descargado más de un aplicativo de delivery en su celular. Esto demuestra que es un consumidor que busca información, ofertas y promociones en diferentes aplicativos. Por otro lado, la mayoría de los encuestados $(64,9 \%)$, tienen edades que oscilan entre 18 y 25 años; y, el 17,4\% entre 26 y 30 años. Con el concepto de tecnofobia se pueden segmentar los potenciales clientes para un negocio, ya que establece quiénes tienen más posibilidad de adoptar este tipo de tecnologías a su vida cotidiana (Sinkovics, Stöttinger, et al., 2002); en los resultados anteriormente presentados, se puede establecer que el público objetivo de los aplicativos móviles de delivery de restaurantes son los consumidores entre 18 y 30 años.

En relación con la percepción de seguridad de los consumidores de restaurantes por aplicativos, existía un temor relacionado con la confianza, mayormente por el robo de datos personales, que el producto no llegue o que no cumpla con las especificaciones solicitadas (Cancino et al, 2019). Sin embargo, en el estudio se verificó que el $69 \%$ de los encuestados mencionó que se sentían seguros o totalmente seguros al introducir datos e información personal en los aplicativos de delivery. Esto se debe a que, en el contexto de la pandemia del COVID-19, la percepción de seguridad estuvo mayormente relacionada con el cumplimiento de los protocolos de bioseguridad por parte del aplicativo de delivery (93.5\%). Esto coincide con la creciente preocupación del consumidor peruano $(80 \%)$ por cuidarse a sí mismo, a su familia y amigos a través de los pedidos por el canal m-commerce, ya que se lograba reducir al mínimo el contacto con otras personas (McKinsey, 2020).

Por otro lado, se verificó que las dimensiones de los aplicativos más importantes para los encuestados son la ubicuidad, la personalización del servicio y la riqueza de la información. Sobre la primera dimensión, se obtuvo respuestas diversas, como la preferencia por realizar pedidos desde casa $(37,5 \%)$, reuniones familiares o sociales $(30,6 \%)$, centro laboral $(21,8 \%)$ y centro de estudios (10,1\%); esto establece que los 
aplicativos de delivery ofrecen ubicuidad, ya que se pueden realizar pedidos desde diferentes lugares. Sobre la personalización del servicio, el $87,8 \%$ de encuestados estuvo de acuerdo y totalmente de acuerdo con la afirmación de que la interfaz de los aplicativos de delivery es amigable; lo que demuestra que los aplicativos se adaptan a las necesidades y requerimientos de cada tipo de cliente, para ofrecerle un servicio más personalizado. Esto genera una conexión con el cliente similiar a la que se da en el canal físico (Laudon y Guercio, 2013). Asimismo, con respecto a la riqueza de la información, el $64,4 \%$ de encuestados estuvo de acuerdo y totalmente de acuerdo con la afirmación de que las comunicaciones que reciben de los aplicativos de delivery generan un aumento en su frecuencia de compra por este medio.

Respecto a la experiencia en el restaurante se deben considerar tres aspectos: la calidad del producto, la calidad del servicio y un amigable entorno físico (Ryu et al, 2012). Sin embargo, en el contexto del servicio de delivery, los restaurantes tienen mayores dificultades para ofrecer la mejor experiencia para los consumidores, ya que el traslado del producto y el servicio son factores tercerizados, que generalmente dependen del aplicativo y su personal. En base a la investigación realizada, se obtuvo información que la mayoría de encuestados (66\%) consideraba que la calidad de la comida era el aspecto más importante de la experiencia gastronómica que ofrecen los restaurantes. Por otro lado, la mayoría de encuestados $(76,1 \%)$ eligió a los restaurantes de comida rápida.

A partir del análisis de la prueba Chi Cuadrado y de los coeficientes de contingencia se obtuvo que las dimensiones con una relación de dependencia y con el mayor nivel de asociación fueron las siguientes: el "ingreso promedio mensual de los encuestados" y "el ticket de promedio de consumo por restaurante", con un nivel de asociación moderadamente alto $(0,498)$, es decir, los peruanos durante la pandemia centraron su preocupación en sus familiares y por ello, buscaron la opción más segura en términos de prevención del contagio por el COVID-19 al momento de solicitar comida de restaurantes a través del delivery; pero a su vez, recortaron algunos gastos, es por eso que en esta correlación no se observa que: usuarios con bajo ingreso gasten más en delivery, ya que es proporcional (McKinsey, 2020).

Asimismo, se verificó un nivel de asociación moderadamente alto $(0,409)$ entre la realización de pedidos delivery más de 1 vez a la semana y el hecho de tener descargado más de un aplicativo. Tal hecho corrobora el crecimiento del canal del m-commerce en el Perú y sobretodo de aplicaciones de delivery, que se han adaptado a las necesidades del consumidor por lo cual el tiempo promedio de navegación en la actualidad es de 19 minutos por día (antes era $16 \%$ menos) (Datum Internacional, 2020). Con el reinicio de las actividades económicas el consumidor se inclinó por aplicaciones que le brindaron una buena experiencia (Datum Internacional, 2020) y por aquellas con una buena reputación, que además consideren factores como: la política de protección al consumidor y de retorno de productos, celeridad en la atención, entre otros (Pan y Zinkhan, 2016).

En contraste, las dimensiones edad del encuestado e impacto de las comunicaciones del aplicativo de delivery en la frecuencia de compra del usuario, obtuvo el menor nivel de asociación, con un valor de 0.251. Esto se relaciona con que los aplicativos generalmente no logran que los clientes sientan una interacción real a través de sus comunicaciones. Por lo tanto, para aumentar la frecuencia de compra los aplicativos de delivery, deberían personalizar en mayor medida sus comunicaciones, ya que, cuando los usuarios perciben una interacción humana real, aumenta la confianza en el canal m-commerce y la posibilidad de que realicen transacciones a través de este canal (Gefen y Straub, 2004).

Finalmente, el análisis de relaciones de dependencia de las dimensiones permitió establecer características relevantes del perfil del consumidor digital de aplicativos móviles en el Perú, como por ejemplo que, el ingreso promedio del consumidor es directamente proporcional con el ticket de consumo que tiene en los locales, también que, existe una relación considerable 
entre aquellos consumidores que piden delivery más de 1 vez a la semana, y aquellos que tienen descargado más de un aplicativo. Esto coincide con lo establecido en base a la teoría de Abuladze y Todua (2011), sobre que el perfil del consumidor de los aplicativos de restaurantes en Lima, Perú está compuesto mayormente de compradores de gangas, ya que, si bien existe una relación entre su ingreso y el ticket de consumo, esto se debe principalmente a una mayor frecuencia de compra a través de diferentes aplicativos, que ofrecen promociones $y$ descuentos. Por otro lado, las comunicaciones de los aplicativos no ejercen un impacto significativo en los consumidores sin importar la edad, y el lugar de uso de los aplicativos tampoco guarda una relación considerable con la ocupación o labor del consumidor.

\section{CONCLUSIONES}

En el presente estudio se verificó que el consumidor de los aplicativos de restaurantes en Lima, Perú está compuesto mayormente del perfil de comprador de gangas (Abuladze y Todua, 2011) e integrante de la generación millenial y parte de la generación Z. A esos consumidores le gustan las ofertas y promociones de sus aplicativos de delivery, también, que tienen descargado más de un aplicativo con el fin de encontrar más variedad de restaurantes y descuentos.

Respecto al comportamiento del consumidor de restaurantes por aplicativos de delivery se verificó la importancia de las dimensiones psicológica, social y personal. Sobre la psicológica, se establece que, si bien los individuos se sentían seguros al introducir datos e información personal en los aplicativos de delivery a su vez en el contexto de la pandemia del COVID-19, existe un temor relacionado al cumplimiento de los protocolos de bioseguridad por parte del aplicativo al momento de la entrega del producto. A raíz de la crisis, ha surgido una "nueva seguridad" que se enfoca en el cumplimiento de los protocolos de bioseguridad debido a la pandemia por el COVID-19, que ha creado un consumidor más exigente $\mathrm{y}$ desconfiado. En relación con la dimensión social, se verifica que el consumidor es influenciable por sus familiares o amigos para realizar pedidos de comida por aplicativos de delivery. Por último, sobre la dimensión personal, se observó una relación considerable entre el nivel de ingreso promedio mensual con el ticket promedio de consumo que gastan en los restaurantes.

Además, se pudo determinar las dimensiones más importantes de los aplicativos para el consumidor de restaurantes por aplicativos de delivery que fueron: la ubicuidad, la personalización del servicio y la riqueza de la información. En ese sentido, es importante señalar que los teléfonos celulares permiten a las personas acceder a todo tipo de información cuando lo deseen y sin la necesidad de una conexión física a internet (Shuang Chen et al., 2011). Luego, se señala la importancia de la personalización del servicio de los aplicativos al desarrollar interfaces amigables que se adaptan a los requerimientos de los clientes buscando imitar el servicio que se ofrece en un canal físico. Sobre la riqueza de la información, que se relaciona con la influencia de las comunicaciones de los aplicativos en la frecuencia de compra de los consumidores. Esto se comprobó con el análisis de correlación que mostró un nivel de asociación moderadamente bajo, principalmente porque los aplicativos no logran un nivel alto de personalización en sus comunicaciones con sus consumidores.

También, se pudo establecer el nivel de relevancia de la prestación del servicio de los restaurantes para los consumidores de Lima en el 2020 que, en el contexto de la pandemia del COVID-19, tiene a la calidad de la comida como el aspecto más importante de la experiencia gastronómica que brindan los restaurantes. La información obtenida contrasta con lo propuesto por Kim et al. (2018) que mencionó que, en la actualidad, los consumidores no solo están buscando productos y servicios, sino una experiencia completa.

Como aportes del presente estudio, se verifica que: (a) la ubicuidad, personalización del servicio y riqueza de la información son las dimensiones en las que se deben enfocar los aplicativos de delivery de restaurantes; (b) en relación con la variable comportamiento del 
consumidor, la dimensión psicológica muestra el surgimiento de una "nueva seguridad", que se relaciona con las entregas de los pedidos y el cumplimento de los protocolos de bioseguridad. Luego, se verifica la importancia para los aplicativos de delivery de restaurantes, que se enfoquen en cuidar sus procesos de entrega de productos.

Por último, se sugiere la realización del mismo estudio en un contexto post pandemia y realizar una comparación con los datos obtenidos en la presente investigación. Además, se verifica que la cobertura del estudio puede ser ampliada no solo a nivel nacional sino también a nivel Latinoamérica, para poder hacer una comparación de los tipos de consumidores que existen en los diferentes países y, sus necesidades específicas. Finalmente, se recomienda analizar las motivaciones de compra de otras generaciones de consumidores como la generación $\mathrm{X}$ o baby boomers, para establecer las diferencias en el uso de los aplicativos por delivery de los restaurantes.

\section{REFERENCIAS}

Abuladze, R., Todua, N. (2011). Internet Marketing. - Estados Unidos: Universal Inc.

Ashraf, A.R., Thongpapanl, N., Menguc, B. y Northey, G. (2016). The Role of M-commerce Readiness in Emerging and Developed Markets [El papel de la preparación para el comercio móvil en los mercados emergentes y desarrollados]. Sage https://doi.org/10.1509/jim.16.0033

Bergström, H., Roback, L., Steinbernreiter, K. (2015). "It's the future!" A qualitative study exploring Generation Y's attitude towards mcommerce. [iEs el futuro! Un estudio cualitativo explorando las actitudes de la generación Y hacia el comercio móvil] Obtenido de http://lnu.divaportal.org/smash/get/diva2:824113/FULLTEXT 01.pdf

Bitner, M.J. (1992). Servicescapes: The impact of Physical Surroundings on Customers and Employees. [El impacto del entorno físico en clientes y empleados]. Journal of Marketing. Obtenido de https://yuiworld.files.wordpress.com/2011/07/6- the-impact-of-physical-surrounding-oncustomers-and-employees.pdf

Cámara Peruana de Comercio Electrónico (2019). Reporte oficial de la industria e-commerce en Perú 2009-2019. Obtenido de https://www.inteligenciaparanegocios.co m/wp- content/uploads/2020/02/Reporte-Oficialde-la-Industria-Ecommerce-en-Peru.pdf

Cancino, M., Huancayo, M., Romero, W., Saavedra, J. (2018) El consumidor del sector moderno y la compra electrónica en Lima y zona norte del Perú. Obtenido de https://repositorio.esan.edu.pe/bitstream/handle/2 0.500.12640/1453/2018_MATPTRU_141_05_T.pdf?sequence $=1 \&$ isAllowed $=\mathrm{y}$

Clarke III, I. (2001). Emerging Value Propositions for M-commerce. [Propuestas de valor emergentes para el comercio móvil]. Journal of Business Strategies. Ebsco. Obtenido de http://search.ebscohost.com/login.aspx?direct=tr $\mathrm{ue} \& \mathrm{db}=\mathrm{bth} \& \mathrm{AN}=36541869 \&$ lang $=$ es $\&$ site $=$ eho st-live

Datum Internacional (2020). Comportamiento online ante coyuntura COVID-19. Obtenido de https://www.datum.com.pe/new_web_files/files/ pdf/2020\%20Comportamiento\%20online\%20ant e\%20coyuntura\%20Covid-19.pdf

Du S., Li, H. (2019). The Knowledge Mapping of Mobile Commerce Research: A visual analysis based on I-Model [El Mapeo del Conocimiento de la Investigación del Comercio Móvil: un análisis visual basado en I-Model]. Sustainability. https://doi.org/10.3390/su11061580

El Peruano (2020). Gobierno incrementa aforo en tiendas y restaurantes en fase 4 de reactivación. Obtenido de https://elperuano.pe/noticia/109792gobierno-incrementa-aforo-en-tiendas-yrestaurantes-en-fase-4-de-reactivacion

Gefen, D., Straub, D. (2004). Consumer trust in B2C e-commerce and the importance of social presence: Experiments in e-Products and eServices. Omega. Science Direct. https://doi.org/10.1016/j.omega.2004.01.006 
Hafi, M.A. (2020). Introduction of a new digital technology in the m-commerce industry. [Introducción de una nueva tecnología digital en la industria del comercio móvil] Obtenido de http://lup.lub.lu.se/student-

papers/record/9019447

Hubert, M., Blut, M., Brock, C., Backhaus, C. y Eberhardt T. (2017). Acceptance of SmartphoneBased Mobile Shopping: Mobile Benefits, Customer Characteristics, Perceived Risks, and the Impact of Application Context. [Aceptación de las compras móviles basadas en teléfonos inteligentes: beneficios móviles, características del cliente, riesgos percibidos e impacto del contexto de la aplicación] Ebsco. https://doi.org/10.1002/mar.20982

Hernández, R., Fernández, C., Baptista, P. (2014). Metodología de la Investigación. McGraw Hill. Mexico

Ipsos Perú (2019a). Estudio sobre el

Comprador en Línea en el Perú. Obtenido de

https://marketingdata.ipsos.pe/user/miestudio/25 50

Ipsos Perú. (2019b). Hábitos, usos y actitudes hacia el smartphone. Obtenido de https://marketingdata.ipsos.pe/user/miestudio/25 51

Ipsos Perú. (2020).Perfil de los peruanos conectados. Obtenido de https://www.ipsos.com/sites/default/files/ct/publi cation/documents/2020-

10/perfil_de_los_peruanos_conectados_0.pdf

Jiménez Martínez, J. y Martín De Hoyos, M.J. (2007) Indicadores y dimensiones que definen la actitud del consumidor hacia el uso del comercio electrónico. Ebsco. Obtenido de http://search.ebscohost.com/login.aspx?direct=tr ue $\& \mathrm{db}=$ fua $\& A N=28566047 \&$ lang=es\&site=eho st-live

Jiménez, N., San Martin, S. y Azuela, J.I. (2016). Trust and satisfaction: the keys to client loyalty in mobile commerce. [La confianza y la satisfacción: claves para la lealtad del cliente en el comercio móvil] Riubu. Obtenido de https://riubu.ubu.es/bitstream/handle/10259/5289 /Jimenezarla_2016.pdf? sequence $=1 \&$ isAllowed $=$

Jones, M., Reynolds, K., Weun, S., Beatty, S. (2003). The product-specific nature of impulse buying tendency [La naturaleza específica del producto en la tendencia de compra impulsiva] Research Gate. https://doi.org/10.1016/S01482963(01)00250-8

Josiam, B.M., Baldwin, W. y Malave, R. (2014). Assessing quality of food, service and customer experience at a restaurant: the case of a studentrun restaurant in the USA. [Evaluación de la calidad de la comida, el servicio y la experiencia del cliente en un restaurante: el caso de un restaurante dirigido por estudiantes en Estados Unidos] Research Gate. Obtenido de https://www.researchgate.net/publication/263927 162_Assessing_quality_of_food_service_and_cu stomer_experience_at_a_restaurant_The_case_of _a_student-run_restaurant_in_the_USA

Kim, E., Beckman, S.L., (2018). Design Roadmapping in an Uncertain World: Implementing a CustomerExperience-Focused Strategy. [Diseño de hoja de ruta en un mundo incierto: implementación de una estrategia centrada en la experiencia del cliente] Sage. Obtenido de https://www.learnefqm.com/wpcontent/uploads/2020/06/article-3-slide-9.pdf

Kotler P., Armstrong, G. (2017) Fundamentos de marketing. 13a edición. Pearson Education. México Laudon K., Guercio, C. (2013) Ecommerce: Negocios, tecnología, sociedad. Pearson Education. México

Laroche, M., McDougall, G., Bergeron, J., Yang, Z., (2004). Exploring How Intangibility Affects Perceived Risk [Explorando cómo la intangibilidad afecta el riesgo percibido]. Sage. https://doi.org/10.1177/1094670503262955

M-commerce en Perú: Valores del 2015-2020. (2020) Euromonitor. Recuperado el 28 de Abril del 2021 de https://www.euromonitor.com 
McKinsey \& Company. (Noviembre de 2020). Survey: Peruvian consumer sentiment during the coronavirus crisis [Encuesta: sentimiento del consumidor peruano durante la crisis del coronavirus]. Obtenido de https://www.mckinsey.com/business-

functions/marketing-and- sales/ourinsights/survey-peruvian-consumer-sentimentduring-the-coronavirus-crisis

McLean, G.; Al-Nabhani, K.; Wilson, A. (2018) Developing a mobile application customer experience model (MACE) - implication for retailers [Desarrollo de un modelo de experiencia del cliente en aplicaciones móviles - implicancia para los retailers]. University of Strathclyde, 1-34. Obtenido de https://strathprints.strath.ac.uk/63220/1/McLean _etal_JBR_2018_Developing_a_mobile_applicat i on_customer_experience_model.pdf

Mercado, K.E., Peréz, C.B., Castro, L.A. y Macias, A. (2019). Estudio Cualitativo sobre el Comportamiento del Consumidor en las Compras en Línea. Scopus. http://dx.doi.org/10.4067/S0718-

07642019000100109

National Restaurant Association (2011). Foundations of restaurant management \& culinary arts. [Fundamentos de gestiónde restaurantes y artes culinarias] Obtenido de https://www.eriesd.org/site/handlers/filedownloa d. ash $x$ ? moduleinstanceid $=23740 \&$ dataid $=36263$ $\&$ FileName=Foundations_Book_Level_I.pdf)

Nayyar, N. (2015). Issues and challenges in ecommerce and m-commerce: a review [Problemas y desafíos en el comercio electrónico y el comercio móvil: una revisión]. International Journal of Current Research. Obtenido de https://www.journalcra.com/sites/default/files/iss ue-pdf/11697.pdf

Pan, Y., Zinkhan G., (2006). Determinants of retail patronage: A meta-analytical perspective. [Determinantes del mecenazgo minorista: una perspectiva meta-analítica]. Journal of Retailing. https://doi.org/10.1016/j.jretai.2005.11.008
Parasuraman, P., Berry, L. y Zeithaml, V.A. (1988). A multiple- Item Scale for measuring consumer perceptions of service quality. Journal of Retailing. Obtenido de https://www.researchgate.net/publication/225083 802_SERVQUAL_A_multiple-

_Item_Scale_for_measuring_consumer_percepti ons_of_service_quality

Rajabion, L. (2015). Critical Factors for Adoption of Mobile Commerce Services. [Factores críticos para la adopción del servicio de comercio móvil]. International Journal of Business and Social Science.http://www.ijbssnet.com/journals/Vol_6 _No_12_December_2015/2.pdf

Ryu, K., Lee, H. and Gon Kim, W. (2012), "The influence of the quality of the physical environment, food, and service on restaurant image, customer perceived value, customer satisfaction, and behavioral intentions". International Journal of Contemporary Hospitality Management. https://doi.org/10.1108/09596111211206141

Schiffman, L., Lazar, L. (2010). Comportamiento del consumidor. Pearson Education. Mexico

Shang, D., Wu, W. (2017) Understanding Mobile Shopping Consumers' Continuance Intention. [Entendiendo la recompra de los consumidores de comercio móvil] Industrial Management and Data Systems. Research Gate. https://doi.org/10.1108/IMDS-02-2016-0052

Shuang Chen, Z., Li, R., Chen, X. y Xu, H.(2011). A Survey Study on Consumer Perception of Mobile- Commerce Applications. [Estudio de una encuesta sobre la percepción del consumidor de las aplicaciones de comercio móvil] Scopus. https://doi.org/10.1016/j.proenv.2011.12.019

Sinkovics, R., Stöttinger, B., Schlegelmilch, B., Ram, S., (2002). Reluctance to use technologyrelated products: Development of a technophobia scale. Thunderbird.

https://doi.org/10.1002/tie.10033

Thihas, J.M., Thangavelan, R. (2018). ECommerce. Shanlax. International Journal of 
Management. https://doi.org/10.5281/

zenodo.1461341

Zhang, P., Benjamin, R. (2007). Understanding information related fields: A conceptual framework [Comprendiendo los campos relacionados con la información: un marco conceptual] Journal of the American Society for information science and technology. 58. 19341947. Obtenido

de https://www.researchgate.net/publication/262407 463_Understanding_information_related_fields_ A_conceptual_framework 